CARDIOVASCULAR MEDICINE

\title{
Diagnostic and prognostic value of ST segment depression limited to the recovery phase of exercise stress test
}

\author{
G A Lanza, M Mustilli, A Sestito, F Infusino, G A Sgueglia, F Crea
}

Heart 2004;90:1417-1421. doi: 10.1136/hrt.2003.031260

See end of article for authors' affiliations

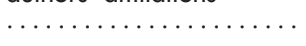

Correspondence to: Dr Gaetana A Lanza, Istituto di Cardiologia, Università Cattolica del Sacro Cuore, L.go A.

Gemelli, 8, 00168 Rome, Italy; g.a.lanza@inwind.it

Accepted 10 March 2004
Objectives: To investigate the diagnostic and prognostic value of ST segment depression limited to the recovery phase of an exercise stress test, as compared with that of ST segment depression appearing during exercise.

Setting: Exercise stress test laboratory of a university hospital.

Patients and design: Clinical and angiographic data were compared for 574 consecutive patients who developed ST segment depression during the active phase of an exercise test (group 1) and for 79 patients who developed ST segment depression only during the recovery phase of the exercise test (group 2).

Results: There were no differences between the two groups in major clinical features. Significant coronary artery stenoses were found in 488 group 1 patients (85\%) and in 62 group 2 patients $(78 \%, p=0.14)$. Three vessel or left main disease was found in $166(29 \%)$ group 1 and in $14(18 \%)$ group 2 patients $(p=0.045)$. At a median follow up of 55.3 months of 321 group 1 and 54 group 2 patients, there were no significant differences in major cardiac events between the groups (univariate relative risk 0.81, 95\% confidence interval 0.25 to $2.68, p=0.72$ ).

Conclusion: The diagnostic and prognostic power of ST segment depression limited to the recovery phase of an exercise test is largely similar to that of ST segment depression induced during effort; thus, assessing ST segment depression during recovery can significantly improve the clinical information derived from exercise stress tests.
$\mathrm{E}$ xercise induced ST segment depression is considered a reliable ECG finding for the diagnosis of obstructive coronary atherosclerosis. ${ }^{12}$ It has also been associated with a worse prognosis for patients with a documented coronary artery disease (CAD)..$^{3-5}$ However, while the diagnostic and prognostic value of ST segment depression occurring during the active phase of exercise test is well recognised, only a few studies have investigated the clinical significance of ST segment depression appearing only during the recovery phase of exercise testing. ${ }^{6-12}$ Thus, in this study we aimed at assessing the diagnostic and prognostic power of recovery-only ST segment depression in a sufficiently large group of consecutive patients who were referred for exercise testing because of suspected or known CAD and who underwent coronary angiography within six months of the stress test.

\section{METHODS \\ Patients}

About 1800 treadmill exercise stress tests are performed each year at our laboratory. Both clinical and exercise data of patients are entered into an electronic database. Among patients who underwent an exercise stress test from 1992 to 1999, we selected those who developed significant ST segment depression (see below) during a symptom limited exercise test and who underwent coronary angiography within six months of the test. Indications for an exercise stress test were diagnostic assessment for suspected angina pectoris, follow up assessment of patients with a known history of CAD (for example, previous angina, myocardial infarction (MI), or coronary revascularisation procedures), and predischarge evaluation of patients admitted for unstable angina. Patients were excluded if they presented with one or more of the following features: recent ( $<6$ months) acute MI; ECG abnormalities at rest that could have interfered with ST segment analysis, including atrial fibrillation, baseline ST segment depression ( $>0.5 \mathrm{~mm}$ ), left bundle branch block, and pacemaker rhythm; and clinical evidence of noncoronary heart disease, including valvar heart disease and cardiomyopathy.

For each patient we obtained information about their age, sex, clinical diagnosis, history of diabetes, previous MI or coronary revascularisation procedures, use of anti-ischaemic drugs, and left ventricular ejection fraction (LVEF) measured by two dimensional echocardiography within three months of the exercise test.

\section{Exercise stress test}

All patients underwent a symptom and sign limited treadmill exercise stress test according to either a Bruce or modified Bruce protocol. Indications for interruption of the test were crescendo angina, ST segment changes $>2 \mathrm{~mm}$ compared with baseline, clinically significant arrhythmias, hypotensive (> $20 \mathrm{~mm} \mathrm{Hg}$ reduction in systolic blood pressure compared with a previous measurement) or hypertensive (systolic blood pressure $>240 \mathrm{~mm} \mathrm{Hg}$ or diastolic blood pressure $>140 \mathrm{~mm} \mathrm{Hg}$ ) response to exertion, and other potentially dangerous clinical conditions (such as arrhythmias or dyspnoea). Three ECG leads (II, V2, and V5) were continuously monitored during the test. The 12 lead ECG was printed and brachial artery cuff blood pressure was recorded before exercise, at the end of each stage during the effort, and at one minute intervals during recovery. The recovery phase of exercise always lasted five minutes, but it was prolonged if clinically indicated. ST segment depression was considered to be significant when it was $\geqslant 1 \mathrm{~mm}$ at 0.08 seconds from the $\mathrm{J}$ point and was either horizontal or downsloping.

Abbreviations: $C A D$, coronary artery disease; $\mathrm{Cl}$, confidence interval; LVEF, left ventricular ejection fraction; MI, myocardial infarction; RR, relative risk 
Table 1 Main clinical data of patients with ST segment depression induced during exercise (group 1) and those with recovery-only ST segment depression (group 2)

\begin{tabular}{llll}
\hline & Group 1 (n= 574) & Group 2 (n=79) & p Value \\
\hline Age (years) & $60(8)$ & $59(9)$ & 0.53 \\
Men & $469(82 \%)$ & $60(76 \%)$ & 0.22 \\
Diabetes & $106(18.5 \%)$ & $13(16.4 \%)$ & 0.66 \\
Previous MI & $296(52 \%)$ & $60(53 \%)$ & 0.79 \\
Unstable angina & $85(15 \%)$ & $11(14 \%)$ & 0.95 \\
LVEF (\%) & $52(8)$ & $52(9)$ & 0.91 \\
PCI & $69(12 \%)$ & $14(18 \%)$ & 0.52 \\
CABG & $41(7 \%)$ & $8(10 \%)$ & 0.35 \\
$\beta$ Blockers & $111(19 \%)$ & $25(32 \%)$ & 0.12 \\
Calcium antagonists & $128(30 \%)$ & $23(37 \%)$ & 0.18 \\
Nitrates & $195(34 \%)$ & $44(56 \%)$ & 0.006 \\
\hline
\end{tabular}

Data are mean (SD) or number (\%)

CABG, coronary artery bypass graft; LVEF, left ventricular ejection fraction; $\mathrm{MI}$, myocardial infarction; $\mathrm{PCl}$, percutaneous coronary intervention.

\section{Coronary angiography}

As an inclusion criterion all patients underwent coronary angiography within six months of the exercise stress test. Performing coronary angiography was at the total discretion of the patients' attending physicians. The presence of coronary stenoses was assessed visually by experienced angiographers. A stenosis in a major epicardial coronary artery was considered to be significant when it was $>50 \%$ of the lumen diameter.

\section{Follow up}

The clinical status of patients was ascertained by telephone interview of patients or their relatives. In case of events, clinical records were checked. As a primary end point we considered the occurrence of cardiac death, resuscitated cardiac arrest, or non-fatal acute MI, whichever occurred first. We regarded as secondary events hospital admission for unstable angina and myocardial revascularisation-that is, percutaneous coronary intervention or coronary artery bypass graft surgery.

\section{Statistical analysis}

Patients were divided into two groups: group l comprised patients with ST segment depression induced during the active phase of the exercise stress test, and group 2 comprised patients with no ST segment changes during exercise but who exhibited a significant ST segment depression during the recovery phase of the test. Discrete variables were compared by $\chi^{2}$ analysis, whereas unpaired Student's $t$ test or MannWhitney U test were used to compare continuous variables, as indicated. Logistic regression was used to evaluate the association of recovery-only, compared with effort induced, ST segment depression with severe (three vessel or left main) CAD.

Survival was analysed by the Kaplan-Meier limit-product method and event-free survival curves were compared by log rank test. Univariate Cox regression analysis was applied to estimate the risk of events associated with recovery-only, compared with exercise induced, ST segment depression. A multivariate Cox regression analysis was also applied to adjust the risk for the major clinical (age, diabetes, previous MI, LVEF, and use of $\beta$ blockers) and exercise (maximum ST segment depression, metabolic equivalents) variables. Data are reported as mean (SD), unless otherwise indicated. A probability value of $\mathrm{p}<0.05$ was always required for significance.

\section{RESULTS}

\section{Baseline clinical characteristics}

A total of 653 patients (529 men and 124 women) fulfilled the selection criteria for the study. Mean (SD) age of the patients was 60 (9) years and 338 (52\%) had a history of MI. Admission to hospital because of unstable angina was the reason for a stress test for 90 patients (14\%). Stable angina, in the absence of previous MI, was the referring diagnosis for 148 patients $(23 \%)$. Overall, $71 \%$ of patients were taking antiischaemic drugs $(21 \% \quad \beta$ blocking agents, $31 \%$ calcium channel blockers, 37\% nitrates). Furthermore, 83 patients (13\%) had undergone percutaneous coronary intervention and $49(7.5 \%)$ coronary artery bypass graft surgery.

Of the 653 patients selected for the study, 574 (88\%) developed ST segment depression during the active phase of the stress test (group 1) and 79 (12\%) exhibited ST segment depression only during the recovery phase of the test (group 2 ). Table 1 shows the main clinical features of the two groups of patients. There were no significant differences between groups with regard to age, sex, history of MI, unstable angina, coronary revascularisation, LVEF, and drug treatment, except for a higher number of patients taking long acting nitrates in group $2(p=0.006)$. The prevalence of recovery-only ST segment depression was similar in patients with $(11.7 \%)$ and in those without $(12.4 \%)$ a previous MI (not significant).

\section{Exercise stress test results}

Table 2 shows the main results of exercise stress tests. There were no differences between the two groups in basal blood pressure and heart rate. At peak exercise, however, heart rate, rate-pressure product, and workload were higher in group 2 patients, whereas maximum ST segment depression was greater in group 1 patients. There was no difference between the two groups in the occurrence of angina.

Table 2 Main results of exercise stress test in the two groups of patients

\begin{tabular}{llll}
\hline & Group $1(\mathbf{n}=574)$ & Group 2 (n=79) & p Value \\
\hline Rest & & & \\
$\quad$ Heart rate (beats $/ \mathrm{min})$ & $75(16)$ & $78(17)$ & 0.24 \\
Systolic blood pressure $(\mathrm{mm} \mathrm{Hg})$ & $140(22)$ & $137(19)$ & 0.15 \\
$\quad$ Rate-pressure product (beats $/ \mathrm{min} \times \mathrm{mm} \mathrm{Hg})$ & $10616(2881)$ & $10633(2900)$ & 0.96 \\
Peak exercise & $122(22)$ & $132(23)$ & 0.0005 \\
$\quad$ Heart rate (beats $/ \mathrm{min})$ & $173(31)$ & $176(32)$ & 0.44 \\
Systolic blood pressure $(\mathrm{mm} \mathrm{Hg})$ & $21330(5979)$ & $23246(6294)$ & 0.008 \\
Rate-pressure product (beats $/ \mathrm{min} \times \mathrm{mm} \mathrm{Hg})$ & $8.3(2.9)$ & 0.0001 \\
$\quad$ METs & $1.9(0.7)$ & $1.3(0.6)$ & $<0.0001$ \\
Maximum ST segment depression $(\mathrm{mm})$ & $53 \%$ & $49 \%$ & 0.31 \\
Angina & & & \\
\hline Data are mean (SD). & & & \\
METs, metabolic equivalents. & &
\end{tabular}


Table 3 Coronary angiographic findings in the two groups of patients

\begin{tabular}{|c|c|c|c|}
\hline & $\begin{array}{l}\text { Group 1 } \\
(n=574)\end{array}$ & $\begin{array}{l}\text { Group } 2 \\
(\mathrm{n}=79)\end{array}$ & p Value \\
\hline Any vessel disease & $488(85 \%)$ & $62(78 \%)$ & 0.14 \\
\hline Number of diseased vessels & & & 0.14 \\
\hline 0 & $86(15 \%)$ & $17(22 \%)$ & \\
\hline 1 & $144(25 \%)$ & $24(30 \%)$ & \\
\hline 2 & $178(31 \%)$ & $24(30 \%)$ & \\
\hline $3 /$ left main & $166(29 \%)$ & $14(18 \%)$ & $0.045^{*}$ \\
\hline \multicolumn{4}{|l|}{ Vessels involved in CAD } \\
\hline LAD & $362(63 \%)$ & $42(53 \%)$ & 0.19 \\
\hline Left circumflex artery & 304 (53\%) & $39(49 \%)$ & 0.55 \\
\hline Right coronary artery & $327(57 \%)$ & $32(40 \%)$ & 0.015 \\
\hline
\end{tabular}

*By logistic regression analysis.

$C A D$, coronary artery disease; $L A D$, left anterior descending coronary artery.

\section{Coronary angiography}

Table 3 summarises the main results of coronary angiography. Significant coronary artery stenoses were found in 488 patients $(85 \%)$ of group 1 and in 62 patients (78\%) of group 2 $(p=0.14)$. Severe CAD (three vessel or left main disease) was more often found in group 1 patients, but it was also detected in a sizeable proportion of group 2 patients $(29 \% \mathrm{v}$ $18 \%$, odds ratio $1.85,95 \%$ confidence interval (CI) 1.01 to $3.44, \mathrm{p}=0.045)$. This difference was largely predicted by the magnitude of maximum ST segment depression during exercise, being no longer significant after correction for this variable (odds ratio $1.64,95 \%$ CI 0.87 to 3.03 ; $p=0.36$ ).

Finally, there was no significant difference between the two groups in the presence of critical stenosis in the left anterior descending artery (63\% in group $1 v 53 \%$ in group 2 , $\mathrm{p}=0.19)$, whereas group 1 had higher prevalence of disease in the right coronary artery (57\% in group $1 \vee 40 \%$ in group 2 , $\mathrm{p}=0.015)$.

\section{Clinical events}

The clinical status of patients at follow up was obtained for 375 patients $(57 \%)$. The duration of follow up ranged from
6-100 months (median 55.3). Of patients who were followed up, 321 belonged to group $1(56 \%)$ and 54 to group $2(68 \%$, $\mathrm{p}=0.036$ ). Median follow up time was 57.4 months (range 6-100) in group 1 and 51.4 months (range 10-90) in group 2 $(p=0.07)$. There were no differences in the major clinical and exercise variables between patients who were and patients who were not followed up (table 4).

Table 5 summarises the number of events occurring in the two groups. According to univariate Cox regression analysis, the relative risk (RR) of major events for group 2, compared with group 1, was 0.81 (95\% CI 0.25 to $2.68, \mathrm{p}=0.72$ ). Furthermore, five year actuarial event-free survival from major cardiac events was similar in the two groups $(95 \%$ group $1 \vee 98 \%$ group 2, p = 0.53) (fig 1). The difference persisted non-significantly after correction for major clinical and exercise variables (RR $0.63,95 \%$ CI 0.17 to 2.27 , $\mathrm{p}=0.48$.

Similarly, there was no significant difference between the two groups in the occurrence of total cardiac events, including both primary and secondary end points (RR 0.78 for group 2 compared with group 1, 95\% CI 0.44 to 1.39 , $\mathrm{p}=0.40$ ). Five year actuarial event-free survival from total cardiac events also did not differ significantly between the two groups (75 in group $1 \vee 77$ in group 2, p $=0.72$; fig 1 ), even after adjustment for major clinical and exercise variables (RR $0.75,95 \%$ CI 0.33 to $1.70, \mathrm{p}=0.49$ ).

\section{DISCUSSION}

Although the diagnostic and prognostic power of exercise induced ST segment depression has been well characterised in several groups of patients, ${ }^{1-5}$ the clinical meaning of ST segment depression limited to the recovery phase of an exercise stress test is poorly understood. Indeed, there are no indications about how to consider this finding in the international guidelines for exercise stress testing. ${ }^{13}$

Our data show that the diagnostic and prognostic power of recovery-only ST segment depression is largely similar to that of ST segment depression induced during the active phase of the test; thus, recovery-only ST segment depression should be carefully assessed when evaluating the results of exercise stress testing in patients with suspected or documented CAD.

\begin{tabular}{|c|c|c|c|}
\hline & Follow up ( $n=375$ ) & No follow up $(n=278)$ & p Value \\
\hline Age (years) & $60.2(9)$ & $59.8(9)$ & 0.60 \\
\hline Men & 305 (81.3\%) & $224(80.6 \%)$ & 0.81 \\
\hline Diabetes & $52(13.9 \%)$ & $37(17.2 \%)$ & 0.59 \\
\hline Previous MI & $181(48.3 \%)$ & $157(56.5 \%)$ & 0.04 \\
\hline Unstable angina & $48(12.5 \%)$ & $48(17.3 \%)$ & 0.27 \\
\hline $\operatorname{LVEF}(\%)$ & $51.3(8)$ & $52.4(9)$ & 0.10 \\
\hline $\mathrm{PCl}$ & $53(14.1 \%)$ & $30(10.8 \%)$ & 0.37 \\
\hline CABG & $31(8.3 \%)$ & $18(6.5 \%)$ & 0.39 \\
\hline \multicolumn{4}{|l|}{ Coronary angiography } \\
\hline 0 vessel CAD & $54(14.4 \%)$ & $50(18 \%)$ & 0.41 \\
\hline 1 vessel CAD & $105(28 \%)$ & $64(23 \%)$ & \\
\hline 2 vessel CAD & $115(30.7 \%)$ & $87(31 \%)$ & \\
\hline 3 vessel CAD & $101(26.9 \%)$ & $77(27.8 \%)$ & \\
\hline$\beta$ Blockers & $76(23.5 \%)$ & $60(26.2 \%)$ & 0.46 \\
\hline Calcium antagonists & $122(32.5 \%)$ & $120(43.1 \%)$ & 0.35 \\
\hline Nitrates & $144(38.4 \%)$ & $95(34.2 \%)$ & 0.36 \\
\hline \multicolumn{4}{|l|}{ Exercise results } \\
\hline Peak heart rate (beats/min) & $124(22)$ & $122(23)$ & 0.31 \\
\hline Peak systolic blood pressure $(\mathrm{mm} \mathrm{Hg})$ & $173(31)$ & $172(31)$ & 0.71 \\
\hline $\begin{array}{l}\text { Peak rate-pressure product } \\
\text { (beats } / \mathrm{min} \times \mathrm{mm} \mathrm{Hg} \text { ) }\end{array}$ & $21768(6066)$ & $21287(6018)$ & 0.32 \\
\hline METs & $6.58(3.0)$ & $6.60(2.9)$ & 0.55 \\
\hline Maximum ST segment depression $(\mathrm{mm})$ & $1.87(0.8)$ & $1.83(0.7)$ & 0.49 \\
\hline Angina & $138(37 \%)$ & $92(34 \%)$ & 0.73 \\
\hline
\end{tabular}


Table 5 Clinical events at follow up

\begin{tabular}{|c|c|c|c|c|}
\hline & Group 1 ( $n=321)$ & Group $2(n=54)$ & Relative risk* $(95 \% \mathrm{Cl})$ & p Value \\
\hline Major cardiac events & $29(9 \%)$ & $3(6 \%)$ & $0.81(0.25$ to 2.68$)$ & 0.72 \\
\hline Cardiac death & $12(2.1 \%)$ & $2(2.5 \%)$ & $1.48(0.33$ to 6.74$)$ & 0.61 \\
\hline Non-fatal MI & $15(5 \%)$ & $1(2 \%)$ & $0.48(0.06$ to 3.62$)$ & 0.47 \\
\hline Resuscitated cardiac arrest & $2(0.3 \%)$ & 0 & NA & NA \\
\hline \multicolumn{5}{|l|}{ Secondary cardiac events } \\
\hline Unstable angina & $28(9 \%)$ & $3(6 \%)$ & 0.87 (0.26 to 2.87 ) & 0.82 \\
\hline $\mathrm{PCl} / \mathrm{CABG}$ & $74(23 \%)$ & $7(13 \%)$ & $0.74(0.34$ to 1.61$)$ & 0.44 \\
\hline Total cardiac events & $131(40.8 \%)$ & $13(24.1 \%)$ & $0.78(0.44$ to 1.39$)$ & 0.40 \\
\hline
\end{tabular}

\section{Prevalence of recovery-only ST segment depression} The prevalence of ST segment depression limited to the recovery phase of exercise test ranges widely among the few studies published in the literature. The variability can probably be explained mainly by differences in the characteristics of the study cohorts and methods applied for positivity of ST segment depression..$^{6-12}$

Among positive exercise tests, quite high proportions of recovery-only ST segment depression have been reported in two studies of asymptomatic, apparently healthy patients: young male aircrew (36\%) in one study ${ }^{6}$ and volunteers of both sexes, with a broad age range $(29 \%)$, in the other. ${ }^{11}$ In contrast, in stable patients with a history of MI (> l year), a lower prevalence of recovery-only ST segment depression was found in two previous studies $\left(6 \%^{7}\right.$ and $\left.4.6 \%{ }^{10}\right)$.

In the present study we observed recovery-only ST segment depression in $12 \%$ of patients with a positive exercise test, a prevalence comparable with that found in two previous studies $(15.5 \%$ and $16 \%)$ of populations of patients similar to ours-that is, with suspected or documented CAD and clinical indications for an exercise test. ${ }^{92}$ Of note, in our study a similar proportion of recovery-only ST segment depression was found in patients with (11.7\%) and in those without $(12.4 \%)$ a previous MI.

The reasons for the appearance of ST depression in the recovery phase, rather than during exercise, are unclear. However, consistently with all previous studies, it cannot be predicted by the clinical characteristics of patients. The relatively frequent occurrence of recovery-only ST segment depression, however, highlights the importance of having an appropriate recovery phase, which should be prolonged to at least five minutes.

\section{Diagnostic value of recovery-only ST segment depression}

In our study, the diagnostic power of recovery-only ST segment depression for significant CAD was similar to that of exercise induced ST segment depression. Significant coronary artery stenoses were found in $85 \%$ of group 1 and $78 \%$ of group 2 patients. Moreover, although severe CAD (three vessel or left main disease) was more prevalent in the group of patients with exercise induced ST segment depression, it was also found in a clinically relevant proportion of patients with recovery-only ST segment depression (18\%).

Our results are comparable with those reported by Lachterman and colleagues ${ }^{9}$ in a smaller cohort of patients clinically similar to ours but consisting only of men. In their study the prevalence of CAD was $87 \%$ among patients with exercise induced ST segment depression and $84 \%$ among patients with recovery-only ST segment depression, with a prevalence of severe CAD in the two groups of $32 \%$ and $19 \%$, respectively. Consistent with these findings are also those reported for different populations of patients: apparently healthy people ${ }^{6}{ }^{11}$ or patients with a previous MI. ${ }^{70}$

\section{Prognostic value of recovery-only ST segment depression}

In our wide population of patients with suspected or documented CAD, who are representative of patients routinely referred for exercise testing, we found that the incidence of cardiac events at a mean follow up of 5.5 years was similar in patients with exertion related and in patients with recovery-only ST segment depression.

Three previous studies assessed only the prognostic value of recovery-only ST depression. Two of these studies, however, concerned apparently healthy people ${ }^{611}$ and the third consisted only of patients with a history of Q wave MI. ${ }^{10}$ All these studies consistently reported a similar prognostic value for exercise induced and recovery-only ST segment depression. Thus, taken together, previous data and ours imply that recovery-only ST segment depression has a valuable clinical meaning independent of a patient's clinical characteristics.

\section{Study limitations}

A limitation of our study is that we obtained clinical follow up data for only $57 \%$ of patients; thus, prognostic assessment actually is a subgroup analysis of our cohort. In fact, there were no major differences in most clinical and exercise variables, including age, LVEF and exercise tolerance,
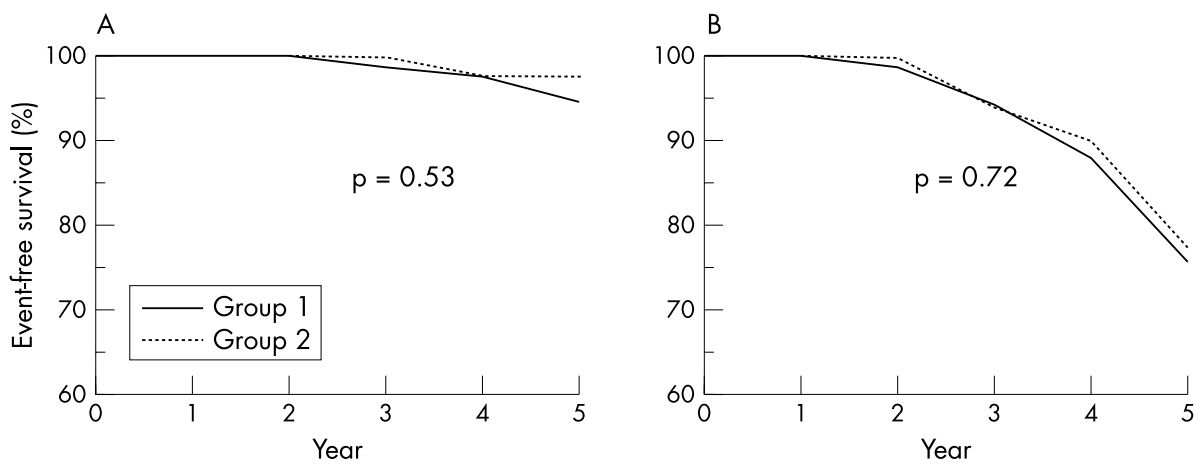

Figure 1 Actuarial event-free survival curves of (A) major cardiac events and (B) total cardiac events in patients with ST segment depression induced during the active phase (group 1) or appearing during the recovery phase (group 2) of an exercise stress test. 
between patients for whom the clinical status could be ascertained at follow up and those for whom it could not be, thus suggesting that the followed up group may be sufficiently representative of the whole population of patients. However, there was a slightly higher prevalence of previous MI in the group of patients who were not followed up, and long term survival from major cardiac events in the group followed up seemed particularly good. Thus, possible selection biases of the followed up patients cannot be excluded.

It should also be considered that most patients were tested while they were being treated with drugs, which might have influenced exercise results. However, it is not known whether any drug can significantly affect the exercise phase (either effort or recovery) during which the ST segment is depressed. Furthermore, there were no relevant differences in drug treatment between the patients with recovery-only and those with exercise induced ST depression.

\section{Conclusion}

In patients with a suspected or documented history of CAD, ST segment depression limited to the recovery phase of an exercise stress test has a diagnostic and prognostic clinical meaning largely comparable with that of ST segment depression induced during the active phase of exercise testing. Thus, careful assessment of recovery-only ST segment depression may add significantly to the clinical information derived from the results of the exercise stress test.

\section{Authors' affiliations}

G A Lanza, M Mustilli, A Sestito, F Infusino, G A Sgueglia, F Crea, Istituto di Cardiologia, Università Cattolica del Sacro Cuore, Rome, Italy

\section{REFERENCES}

1 Gianrossi R, Detrano R, Mulvihill D, et al. Exercise-induced ST depression in the diagnosis of coronary artery disease: a meta-analysis. Circulation 1989;80:87-98.

2 Detrano R, Gianrossi R, Froelicher V. The diagnostic accuracy of the exercise electrocardiogram: a meta-analysis of 22 years of research. Prog Cardiovasc Dis 1989:32:173-206.

3 Weiner DA, Ryan TJ, McCabe $\mathrm{CH}$, et al. Prognostic importance of a clinical profile and exercise test in medically treated patients with coronary artery disease. J Am Coll Cardiol 1984;3:772-9.

4 Mark DB, Hlatky MA, Harrell FE Jr, et al. Exercise treadmill score for predicting prognosis in coronary artery disease. Ann Intern Med 1987; 106:793-800.

5 Morrow K, Morris CK, Froelicher VF, et al. Prediction of cardiovascular death in men undergoing noninvasive evaluation for coronary artery disease. Ann Intern Med 1993:118:689-95.

6 Froelicher VF, Thompson AJ, Longo MR Jr, et al. Value of exercise testing for screening asymptomatic men for latent coronary artery disease. Prog Cardiovasc Dis 1976;18:265-76.

7 Karnegis JN, Matts J, Tuna N, et al. Comparison of exercise-positive with recovery-positive treadmill graded exercise tests. Am J Cardiol 1987;60:544-54

8 Savage MP, Squires LS, Hopkins JT, et al. Usefulness of ST-segment depression as a sign of coronary artery disease when confined to the postexercise recovery period. Am J Cardiol 1987:60:1405-6.

9 Lachterman B, Lehmann KG, Abrahamson D, et al. 'Recovery only' STsegment depression and the predictive accuracy of the exercise test. Ann Intern Med 1990;112:11-6.

10 Casella G, Pavesi PC, Di Niro M, et al. ST sottoslivellato nella fase di recupero del test da sforzo in pazienti stabili con pregresso infarto miocardio. $G$ ltal Cardiol 1996;26:1401-13.

11 Rywik TM, Zink RC, Gittings NS, et al. Independent prognostic significance of ischemic ST-segment response limited to recovery from treadmill exercise in asymptomatic subjects. Circulation 1998:97:2117-22.

12 Soto JR, Watson DD, Beller GA. Incidence and significance of ischemic STsegment depression occurring solely during recovery after exercise testing. Am J Cardiol 2001;88:670-2.

13 Gibbons RJ, Balady GJ, Bricker JT, et al. ACC/AHA 2002 guideline update for exercise testing: summary article. A report of the American College of Cardiology/American Heart Association task force on practice guidelines (committee to update the 1997 exercise testing guidelines). J Am Coll Cardiol 2002;40:1531-40

\section{IMAGES IN CARDIOLOGY}

\section{"Myxomatous" mitral stenosis}

A 78 year old man was admitted because of a sudden appearance of stupor, dysarthria, and left sided hemiplegia. Physical examination revealed an irregular heart rate, an accentuated first heart sound, an "opening snap", and a long apical mid diastolic rumbling murmur. The ECG showed atrial fibrillation. A presumptive diagnosis of mitral stenosis was made on admission. Transoesophageal echocardiography revealed a large left atrial (LA) highly mobile mass (panel A, arrow) attached to the interatrial septum and confined to the left atrium in systole. In diastole (panel B) the tumour (arrow) was seen prolapsing across the mitral valve into the left ventricle (LV) and partially obstructing it, thus producing a "tumour flop" and mimicking valvar mitral stenosis.

Shortly after admission, while awaiting surgery, the patient became comatose. A computed tomographic scan of the brain showed a large infarct in the territory of the right middle cerebral artery. The patient died the next morning. His family refused a postmortem examination.
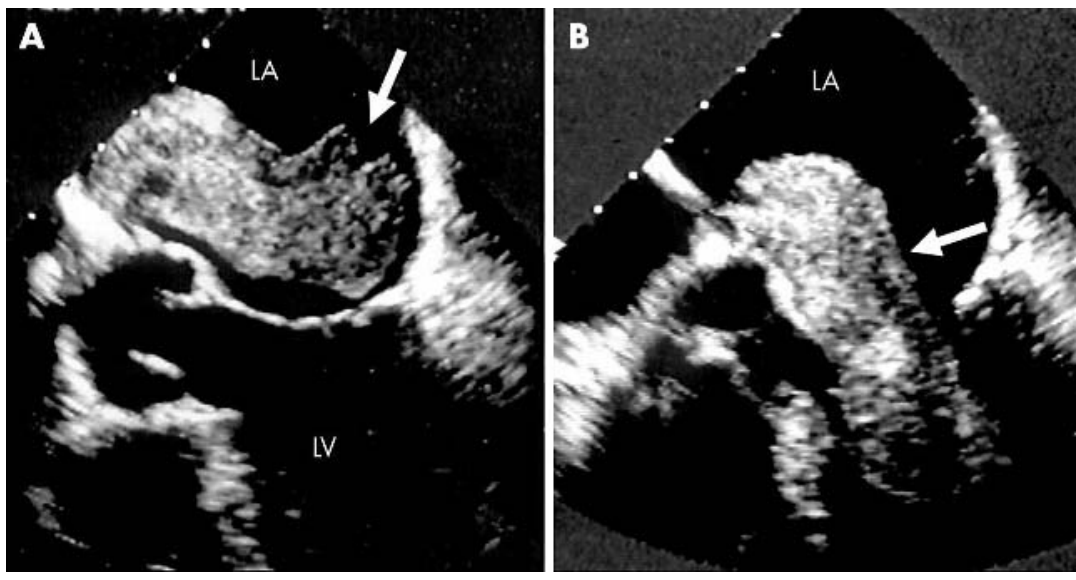

Large mobile left atrial tumours (in this case most likely a myxoma) may mimic valvar mitral stenosis and present as an acute neurological event. This case also explains some of the physical findings in patients with left atrial tumour.

S Atar

L Bloch

T Rosenfeld atar_sh@clalit.org.il 